\title{
Experiments with the drinking bird
}

\author{
J. Güémez ${ }^{a, 1}$, R. Valiente ${ }^{a, 2}$, C. Fiolhais ${ }^{b, 3}$, and M. Fiolhais ${ }^{b, 4}$ \\ ${ }^{a}$ Departamento de Física Aplicada \\ Universidad de Cantabria \\ E-39005 Santander, Spain \\ ${ }^{b}$ Departamento de Física and Centro de Física Computacional \\ Universidade de Coimbra \\ P-3004-516 Coimbra, Portugal
}

\begin{abstract}
We made some experiments with the drinking bird oriented by a quantitative model for its period of oscillation. The effect of humidity on the motion was studied, concluding that there are two evaporation regimes. We made a computer simulation which describes well the drinking bird dynamics.
\end{abstract}

\section{Introduction}

The drinking bird (or dunking duck, dipping bird, etc.), patented in 1946 [1], is not only a toy but also a demonstration apparatus on liquid-vapor equilibrium and evaporation [2]. Figure 1 shows a scheme and a photo of this intriguing thermodynamical device [3, 4]. In Appendix A (Tab. 2) we list geometric and physical data for the toy [5] used in our experiments.

The bird consists of two spherical glass bulbs connected by a glass tube, which enters well inside the lower bulb. The bottom bulb (bird's body, hereafter simply referred to as the body) is almost filled with a highly volatile liquid, normally methylene chloride $\left(\mathrm{CH}_{2} \mathrm{Cl}_{2}\right)$, whose normal boiling point is close to room temperature. There is no air inside the system, only this internal liquid in thermal equilibrium with its vapor.

The top bulb (bird's head, hereafter simply called head) is covered with a porous tissue. It has a small plastic hat and a long beak which is covered with the same tissue as the head. The bird may oscillate around a horizontal metallic bar attached to the middle of the tube. When the bird leans completely forward, it "drinks" water from a glass, although other external liquids may be used as well. We call this a dip.

\footnotetext{
${ }^{1}$ guemezj@unican.es

${ }^{2}$ valientr@unican.es

3 tcarlos@teor.fis.uc.pt

${ }^{4}$ tmanuel@teor.fis.uc.pt
} 
The drinking bird undergoes a cycle, which at first sight may seem a perpetuum mobile. Let us explain qualitatively the oscillations performed by the bird. At the beginning of the cycle the bird is upright, with all the internal liquid in the lower sphere. The water on the head is in contact with its vapor at a given temperature. If the vapor pressure is smaller than its saturation (or equilibrium) value, evaporation occurs spontaneously. This cools the head outside, and therefore the $\mathrm{CH}_{2} \mathrm{Cl}_{2}$ vapor inside also cools. The vapor in the head condenses in very small drops, such that it remains in equilibrium with the internal liquid as the temperature decreases. The vapor pressure inside the head becomes smaller than that in the body according to the Clausius-Clapeyron equation, and this pressure gradient forces the internal liquid to rise up in the tube. As the liquid rises, the center of mass of the system also rises and the momentum produced by the weight eventually forces the bird to tip forward and to dive its beak in the glass, keeping the head wet. When the bird is almost horizontal, the lower end of the tube emerges above the internal liquid surface and some vapor passes from the body to the head (see Fig. 1). While drinking, the bird remains horizontal for a short time. Then, part of the liquid drains back into the body and the bird returns to its upright position. As water evaporation continues in the head, the internal liquid comes up again, starting a new cycle [3]. From the temperature difference between head and body work can be produced so that the drinking bird is in fact a thermal engine. At the most fundamental level, the ability to produce work lies in the difference between chemical potential of the external liquid and of its vapor.

At the most fundamental level, the ability to produce work lies in the difference between the chemical potential of the external liquid and of its vapor. However, note that evaporation on the head is not necessary for the drinking bird to work. A temperature gradient between body and head may simply be obtained by heating the body, e.g. illuminating a black painted body with a light bulb or solar light [4]. Our experiments with the "sunbird" (drinking bird which does not drink) are described in a separate paper [6].

Motivated by the fact that we did not find quantitative descriptions of the drinking bird in textbooks, journals or in the internet, we present in this paper a model which relates its period, i.e. the time between consecutive dips, to some properties of the internal and external liquids and to the bird's dimensions. We performed various experiments with a drinking bird, aiming at checking our model. One of them is on the humidity dependence of the water drinking bird. Furthermore, we developed a computer simulation of the bird's dynamics.

In Sec. 2 we present our model and compare it with experiment, using various liquids as cooling agents. In Sec. 3 we analyze the influence of humidity on the period when water 
is used as external liquid. In Sec. 4 we study the system dynamics and report the results of the numerical integration of the equations of motion. The conclusions are given in Sec. 5. In two Appendixes we indicate the characteristics of the drinking bird and evaluate its moment of inertia and torque, which are needed in Sec. 4.

\section{Period of oscillation}

The cooling of the head with respect to the body, during one period, is directly related to the evaporation of a certain mass of external liquid outside the head, which we denote by $\Delta m_{\mathrm{E}}\left(\Delta m_{\mathrm{E}}>0\right)$. The corresponding temperature decrease inside the head during one cycle, $\Delta T$, is such that

$$
C \Delta T=-\Delta m_{\mathrm{E}} \Delta h_{v}^{\mathrm{E}},
$$

where $C$ is some effective heat capacity of the head, which is a characteristic of a given bird, and $\Delta h_{v}^{\mathrm{E}}$ is the specific evaporation enthalpy of the external liquid. The right hand side of Eq. (1) is the energy loss in one period, which we relate, through that equation, to the temperature decrease inside the head during one period. The minus sign in Eq. (1) makes $\Delta T$ a negative quantity.

On the other hand, according to the Clausius-Clapeyron equation [7], the temperature decrease $\Delta T$ is related to the pressure decrease inside the head in one period, $\Delta P$, by

$$
\Delta T=\frac{\Delta P}{B},
$$

with

$$
B=\frac{\Delta h_{v}^{\mathrm{I}} P_{v}^{\mathrm{I}}\left(T_{\mathrm{R}}\right)}{R T_{\mathrm{R}}^{2}}
$$

where $\Delta h_{v}^{\mathrm{I}}$ is the molar vaporization entalphy variation of $\mathrm{CH}_{2} \mathrm{Cl}_{2}, P_{v}^{\mathrm{I}}\left(T_{\mathrm{R}}\right)$ the vapor pressure at room temperature, $T_{\mathrm{R}}$, and $R$ is the ideal gas constant. Equation (2) is only valid if $\Delta T$ is small enough.

At any time, the pressure difference between head and body (a negative quantity) is given by $-\rho_{\mathrm{I}} g z$, where $\rho_{\mathrm{I}}$ is the internal liquid density and $z$ is the height of the level of the internal liquid in the tube with respect to its surface level in the body. The total pressure decrease inside the head in one period (a negative quantity) is given by

$$
\Delta P=-\rho_{\mathrm{I}} g \Delta z
$$

where $\Delta z$ is the $z$ variation in one period. Inserting this expression in Eq. (2) the temperature variation in one period is

$$
\Delta T=-\frac{\rho_{\mathrm{I}} g \Delta z}{B} .
$$


Of course, a similar relation holds between any variation of $z$ and the corresponding variation of $T$ (provided the variations are small).

If the evaporation rate of the external liquid (a negative quantity), $\dot{m}_{\mathrm{E}}$, is approximately constant, the period is

$$
\tau=-\frac{\Delta m_{\mathrm{E}}}{\dot{m}_{\mathrm{E}}}=\frac{C \Delta T}{\dot{m}_{\mathrm{E}} \Delta h_{v}^{\mathrm{E}}},
$$

where we have used Eq. (1). The numerator $C \Delta T$ is a characteristic of a drinking bird operating with a given external liquid. Although the evaporation rate does not remain constant for many external liquids (it changes due to the structure of the felt that covers the head) it may still be considered constant for small time intervals (say, a few periods — see, below, Fig. 3 and Tab. 1) so that Eq. (6) holds.

In order to verify Eq. (6) we measured periods and evaporation rates not only for water - the usual liquid drunk by the bird - but also for other liquids whose partial pressure in the air is zero. In fact, when water is the external liquid, the air humidity directly affects the bird's dynamics: the period is longer in humid days than in dry ones. For very high humidities, the toy does not even work. Clearly, this problem does not occur for other liquids.

We observed that the period, excluding the short time taken by the dip, is the time needed for the internal liquid to reach the top of the tube and fill approximately half of the head: $z_{\max } \approx L+d_{\mathrm{i}}^{\mathrm{H}} / 2$. We also observed that, after a dip, the internal liquid does not completely return to the body, the tube being half filled when a new cycle starts: $\Delta z_{0}=L / 2$. In Fig. 2 (a) we show the dependence of $z$ with time obtained in a simulation of the bird's motion whose details will be given only in Sec. 4. In Fig. 2 (b) we show the temperature variation (obtained in the same simulation), which is related to the $z$ variation through Eq. (5). After the dip the temperature inside the head only rises to $T_{\mathrm{R}}-\left|\Delta T_{0}\right|$ and along the cycle it drops $|\Delta T|$ (with $\left|\Delta T_{0}\right| \approx|\Delta T|$ ).

For our bird (see Tab. 2) we have $B=(2.07 \pm 0.01) \times 10^{3} \mathrm{PaK}^{-1}$. This value is obtained inserting in Eq. (3) $\Delta h_{v}^{\mathrm{I}}=28094.50 \mathrm{~J} \mathrm{~mol}^{-1}, R=8.3145 \mathrm{~J} \mathrm{~K}^{-1} \mathrm{~mol}^{-1}$, and $T_{\mathrm{R}}=(22.5 \pm 0.1){ }^{\circ} \mathrm{C}$. The liquid-vapor pressure of the internal liquid $P_{v}^{\mathrm{I}}\left(T_{\mathrm{R}}\right)$ in Eq. (3) is obtained from the solution of the Clausius-Clapeyron equation:

$$
P_{v}^{\mathrm{I}}\left(T_{\mathrm{R}}\right)=P_{0} \exp \left[-\frac{\Delta h_{v}^{\mathrm{I}}}{R}\left(\frac{T_{\mathrm{b}}^{\mathrm{I}}-T_{\mathrm{R}}}{T_{\mathrm{R}} T_{\mathrm{b}}^{\mathrm{I}}}\right)\right] .
$$

For $P_{0}=1.013 \times 10^{5} \mathrm{~Pa}$ (normal atmospheric pressure) and $T_{\mathrm{b}}^{\mathrm{I}}=313.15 \mathrm{~K}$ (normal boiling point of the internal liquid, which is indeed close to $\left.T_{\mathrm{R}}[4]\right)$ one gets $P_{v}^{\mathrm{I}}\left(T_{\mathrm{R}}\right)=(0.535 \pm$ 
$0.002) \times 10^{5} \mathrm{~Pa}$. For our bird we measured $\Delta z=(4.2 \pm 0.1) \mathrm{cm}$, so that from eqs. (4) and (5), $\Delta P=-(5.5 \pm 0.1) \times 10^{2} \mathrm{~Pa}$ and $\Delta T=-(0.266 \pm 0.005){ }^{\circ} \mathrm{C}$. This $\Delta T$ is indeed small in comparison with $T_{\mathrm{b}}^{\mathrm{I}}-T_{\mathrm{R}}$, so that Eq. (2) is valid. In a similar fashion, the $\Delta P$ is small when compared to $P_{v}^{\mathrm{I}}$. These values for $\Delta T$ and $\Delta P$ are specific of a given drinking bird. A small $\Delta T$ (and, therefore, a small $\Delta P$ ) facilitates the operation of the drinking bird.

To measure evaporation rates, we placed our drinking bird on a digital balance (whose precision is $\pm 0.001 \mathrm{~g}$ ). After pouring a few drops of different liquids on the head, we measured the time evolution of both the mass of external liquid and the period ${ }^{5}$. Note that in these experiments the bird does not drink when it dips, so that the external liquid on the head that evaporates is not replaced by a new one. Figure 3, which presents results for ethylic alcohol, allows us to confirm the inverse proportionality between period and evaporation rate indicated by Eq. (6).

\begin{tabular}{|c|c|c|c|c|c|}
\hline & $\begin{array}{l}\Delta h_{v}^{\mathrm{E}} / \\
\mathrm{J} \mathrm{g}^{-1}\end{array}$ & $\begin{array}{c}t / \\
\min \end{array}$ & $\begin{array}{c}\dot{m}_{\mathrm{E}} \times 10^{4} \\
\mathrm{~g} \mathrm{~s}^{-1}\end{array}$ & $\begin{array}{c}\tau / \\
\mathrm{s}\end{array}$ & $\begin{array}{c}C \Delta T \\
\mathrm{~J}\end{array}$ \\
\hline \multirow[t]{2}{*}{$\mathrm{CH}_{3} \mathrm{Cl}$} & 247.021 & 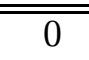 & -21.6 & $2 \pm 0.5$ & $=-1.1 \pm 0.3$ \\
\hline & & 3 & -4.0 & $10 \pm 0.5$ & $-0.99 \pm 0.05$ \\
\hline $\mathrm{C}_{6} \mathrm{H}_{14}$ & 330.757 & 0 & -19.6 & $1.5 \pm 0.5$ & $-1.0 \pm 0.3$ \\
\hline \multirow[t]{2}{*}{$\mathrm{C}_{4} \mathrm{H}_{8} \mathrm{O}_{2}$} & 368.438 & 0 & -5.67 & $5 \pm 0.5$ & $-1.04 \pm 0.13$ \\
\hline & & 6 & -2.33 & $12.5 \pm 0.5$ & $-1.07 \pm 0.04$ \\
\hline \multirow[t]{2}{*}{$\mathrm{C}_{2} \mathrm{H}_{6} \mathrm{O}$} & 841.547 & 0 & -4.40 & $3 \pm 0.5$ & $-1.1 \pm 0.2$ \\
\hline & & 10 & -0.90 & $12.5 \pm 0.5$ & $-0.95 \pm 0.04$ \\
\hline \multirow[t]{2}{*}{$\mathrm{CH}_{4} \mathrm{O}$} & 1101.128 & 0 & -5.91 & $1.5 \pm 0.5$ & $-1.0 \pm 0.3$ \\
\hline & & 7 & -0.96 & $9.5 \pm 0.5$ & $-1.00 \pm 0.05$ \\
\hline $\mathrm{H}_{2} \mathrm{O}$ & 2257.104 & 0 & -0.644 & $9 \pm 0.5$ & $-1.30 \pm 0.07$ \\
\hline$(H=50 \%)$ & & 45 & -0.541 & $10 \pm 0.5$ & $-1.22 \pm 0.06$ \\
\hline
\end{tabular}

Table 1: For several external liquids listed are the specific evaporation enthalpies $\left(\Delta h_{v}^{\mathrm{E}}\right)$ [8], the times, the evaporation rates, the periods, and $C \Delta T=\dot{m}_{\mathrm{E}} \tau \Delta h_{v}^{\mathrm{E}}$. From top to bottom, the liquids are: chloroform, n-hexane (its evaporation is so quick that, after 1 minute, the bird stops), ethyl acetate, ethylic alcohol, methylic alcohol, and water at $50 \%$ humidity.

Table 1 displays the evaporation enthalpies for various external liquids as well as our measured quantities (time, evaporation rate and period) using different external liquids. We note that the evaporation rate of water is lower than for the other liquids and, consequently, the initial period is much larger. But an interesting fact is that $C \Delta T=\dot{m}_{\mathrm{E}} \tau \Delta h_{v}^{\mathrm{E}}$

\footnotetext{
${ }^{5}$ The time interval between consecutive dips experimentally measured should be corrected for the time elapsed while the bird is at rest in the horizontal position (approximately $1 \mathrm{~s}$ ) in order to be compared with Eq. (6).
} 
is approximately constant for a given drinking bird operating with a given external liquid. Moreover, it turns out that, for liquids with lower evaporation enthalpies (the organic liquids in Tab. 1, which are also less dense than water), that constant is the same for all of them: $C \Delta T \approx-1 \mathrm{~J}$. Since $\Delta T$ is known from Eq. (5), one may extract the value of $C$. The partial pressure of all organic liquids in the air is zero, and the lower the evaporation enthapies the higher the evaporation rates. On the other hand, for water $C \Delta T \approx-1.26 \mathrm{~J}$, so that $C=4.75 \mathrm{~J}^{\circ} \mathrm{C}^{-1}$, a value we are going to use in Sec. 4 .

\section{Influence of humidity}

As already mentioned, air humidity affects the bird's period, if water is used as external liquid. At room temperature, when the vapor partial pressure, $P^{\mathrm{E}}$, is equal to the liquidvapor equilibrium pressure at that temperature, $P_{v}^{\mathrm{E}}\left(T_{\mathrm{R}}\right)$, the water on the head does not evaporate anymore and the bird stops. If the humidity is close to saturation, the evaporation rate is low and the periods are large. On the other hand, low partial pressure of water in dry days leads to a large evaporation rate and short periods.

According to Fick's law, the evaporation rate of water is given by $[9,10]$

$$
\dot{m}_{\mathrm{E}}=-\eta_{0}(100-H)
$$

where $\eta_{0}>0$ is a diffusion coefficient depending on the substance and on the evaporation area and $H=100 P^{\mathrm{E}} / P_{v}^{\mathrm{E}}\left(T_{\mathrm{R}}\right)$ is the relative humidity ${ }^{6}$. Inserting Eq. (8), which is valid for normal convection, in Eq. (6), the period becomes $\tau=\kappa_{0}(100-H)^{-1}$, where $\kappa_{0}=$ $\Delta m_{\mathrm{E}} / \eta_{0}$. However, due to the motion of the bird, this equation is not exactly observed. Our experimental results (described below) suggest a different power dependence of the period on the humidity, namely

$$
\tau(H)=\kappa(100-H)^{-\beta},
$$

where $\kappa$ and $\beta$ are phenomenological parameters.

In order to study the evaporation rate we placed the drinking bird in a closed chamber (Fig. 4) and measured the periods (in seconds) for various relative humidities. Figure 4 also shows the logarithm of the period as a function of the logarithm of $100-H$. The experimental results clearly show two linear dependences of $\ln \tau$ with $\ln (100-H)$ in two different ranges of the humidity, validating Eq. (9). Up to $\ln (100-H)=3.2$, i.e.

\footnotetext{
${ }^{6}$ For water at room temperature, the value $P_{v}^{\mathrm{E}}\left(T_{\mathrm{R}}\right)=3.26 \times 10^{3} \mathrm{~Pa}$ is found from the Clausius-Clapeyron equation, inserting $T_{\mathrm{b}}^{\mathrm{E}}=373.15 \mathrm{~K}$ and $\Delta h_{v}^{\mathrm{E}}$ (given in Tab. 1) in Eq. (7) instead of $T_{\mathrm{b}}^{\mathrm{I}}$ and $\Delta h_{v}^{\mathrm{I}}$, respectively.
} 
approximately $75 \%$ relative humidity, the linear fit to the data leads to $\beta=1.82$ and $\ln \kappa=9.44$. We interpret this power law as due to forced air convection. Above that humidity the linear fit to the data yields $\beta=1.24$ and $\ln \kappa=7.69$. Normal evaporation $(\beta=1)$ would only occur if the bird were at rest or slowly moving.

\section{Simulation of the dynamics}

We present now a model for the bird's dynamics based on the previous description of water evaporation. We use as dynamical variable the column height of the internal liquid at time $t, z(t)$.

From eqs. (6) and (9) the mass of water, $\mathrm{d} m_{\mathrm{E}}$, evaporated during the time interval $\mathrm{d} t$ is given by

$$
\mathrm{d} m_{\mathrm{E}}=-\eta(100-H)^{\beta} \mathrm{d} t
$$

where $\eta=\Delta m_{\mathrm{E}} / \kappa$. The values for $\kappa$ and $\beta$ are those found in Sec. 3. From the data of Tab. 1 , one finds $\Delta m_{\mathrm{E}}=6.2 \times 10^{-4} \mathrm{~g}$, so that we have $\eta=4.92 \times 10^{-11} \mathrm{~kg} \mathrm{~s}^{-1}$ for $H<75 \%$ and $\eta=28.3 \times 10^{-11} \mathrm{~kg} \mathrm{~s}^{-1}$ for $H>75 \%$.

The evaporation of mass $\mathrm{d} m_{\mathrm{E}}$ leads to a temperature decrease $\mathrm{d} T$ given by an equation similar to (1): $\mathrm{d} T=-\mathrm{d} m_{\mathrm{E}} \Delta h_{v}^{\mathrm{E}} / C$ (the heat capacity $C$ was obtained in Sec. 2). The temperature decrease $\mathrm{d} T$ in the time interval $\mathrm{d} t$ leads, in turn, to a pressure gradient $\mathrm{d} P$ inside the head given by an equation similar to Eq. (2), i.e. $\mathrm{d} P=B \mathrm{~d} T$. As a consequence, in the time interval $\mathrm{d} t$, the liquid rises in the tube $\mathrm{d} z=-\mathrm{d} P / \rho_{\mathrm{I}} g$. The time evolution of the variable $z$ is given by $z(t+\mathrm{d} t)=z(t)+\mathrm{d} z$, with $z(0)=0$.

With no friction, the angular acceleration is $\alpha=M(z) / I(z)$ where $I(z)$ is the moment of inertia and $M(z)$ the torque with respect to the rotation axis, both depending on the level $z(t)$. The quantities $I(z)$ and $M(z)$ are evaluated in Appendix B.

Since a realistic description of the bird's motion requires frictional effects, we add to the torque a term proportional to the angular velocity, i.e., we define $\mathcal{M}(z)=M(z)-b \omega$, where $b$ is a friction coefficient which is a parameter and $\omega$ the angular velocity. In our model we used $b=7.5 \times 10^{-7} \mathrm{~J} \mathrm{~s}$, though the period is not much sensitive to this parameter. That value was chosen to reproduce the experimental damping.

The angular acceleration $\alpha(t)$ is then given by

$$
\alpha=\frac{\mathcal{M}(z)}{I(z)},
$$


and the angular velocity and the angle between the tube and the vertical direction are given by

$$
\begin{aligned}
\omega(t+\mathrm{d} t) & =\omega(t)+\alpha \mathrm{d} t \\
\theta(t+\mathrm{d} t) & =\theta(t)+\omega(t+\mathrm{d} t) \mathrm{d} t
\end{aligned}
$$

according to the Euler-Cromer algorithm [11] used in our calculation. The time step $0.001 \mathrm{~s}$ was used in the numerical integration of the equations of motion.

When $\theta=90^{\circ}$, the internal liquid partly returns from the head to the body and the angular velocity and height of the internal liquid are set to $\omega=0$ and $z=L / 2$, respectively, before the new cycle starts. Accordingly, the temperature when a new cycle starts is $T_{\mathrm{R}}-\left|\Delta T_{0}\right|$, with $\left|\Delta T_{0}\right|=0.211{ }^{\circ} \mathrm{C}$ calculated from Eq. (5) with $\Delta z_{0}=L / 2$ (see Fig. 2). The value $\Delta T=-0.283{ }^{\circ} \mathrm{C}$ found in our simulation is consistent with that evaluated in Sec. 2 from Eq. (5). Also the maximal $z$ obtained in the simulation agrees with the experimental fact that the head was half filled right before the dip.

Figure 5 (a) shows the angle $\theta$ as a function of time, for the humidity $H=65 \%$ and an initial quasi-vertical and motionless bird. The period is $19.0 \mathrm{~s}$, in agreement with the experimental value $19.5 \pm 0.5 \mathrm{~s}$ obtained from the data fit in Fig. 4 . Figure 5 (b) shows the same quantity for $85 \%$ humidity. Now the period is $74.5 \mathrm{~s}$, close to the experimental value, $76.1 \pm 0.5 \mathrm{~s}$, also obtained from the data fit in Fig. 4. In Fig. 4 the open circles represent the simulation results for the period: the conclusion is that our model reproduces very well the data.

Very often the drinking bird is incorrectly presented as a perpetual motion machine and the graphs of Fig. 5 may cause the same wrong impression that the motion stands forever. In the model we always assume that the head is wet, i.e. there is always external liquid in the head to evaporate. For the drinking bird studied in this paper, the water reservoir was enough to keep it moving for days, so the results of a model that takes into account the exhaustion of water in the reservoir should be presented using a quite different time (horizontal) scale.

There are some effects in the real dynamics of the bird which are not accounted for by our simple model. The cooling mechanism inside the head is admittedly naif; the friction varies with angular velocity probably in a more complicated way than the one we have assumed; the evaporation rate changes with the angular velocity; the internal liquid can not be modeled as a solid, etc. 


\section{Conclusions}

The drinking bird is a thermal engine since it can be used to produce work from a temperature difference. The ability to produce work has its origin in the difference between the chemical potentials of the external liquid and its vapor (Fick's law). There are many thermal engines operating directly by temperature difference (e.g., Carnot and Stirling cycles) or chemical non-equilibrium (internal combustion engines). But there are not many examples in which a chemical potential difference produces work without any chemical reaction $[12]$.

Although the drinking bird is a well-known device, as far as we know only qualitative descriptions of its operation are available in the literature. In this work we presented a quantitative model of the bird's motion. We studied the influence of different external liquids on the bird's behavior, confirming that the period was smaller for more volatile liquids like some organic liquids. Taking water as external liquid, we observed how the period depends on the relative humidity, having found two evaporation regimes: one at low humidities $(H<75 \%)$ and the other at high humidities $(H>75 \%)$. Finally, we have presented a numerical integration of the equations of motion for a bird which drinks water. Our results reproduce well the measured bird's periods. Moreover, this simulation is pedagogically interesting to check the importance of different parameters (mass of water in head, etc.) and to see the role of initial conditions (horizontal or vertical initial position, etc.). It may be applied not only to the drinking bird but also to the sunbird as we shall explain in another paper [6].

\section{Appendix A: Bird's data}

We measured not only the external dimensions of a drinking bird [5] but also the glass thickness of the tube, the head and the body, the masses of the hat, the beak, etc. (we had, therefore, to break a bird). We list the bird's data in Tab. 2.

\section{Appendix B: Bird's moment of inertia and torque}

When the internal liquid reaches the height $z$ inside the internal tube, the moment of inertia $I(z)$ with respect to the rotation axis may be approximated by (for notation, see Tab. 2 and Fig. 1)

$$
I(z)=I_{0}+\left\{\left[V_{\mathrm{I}}-z \pi\left(\frac{d_{\mathrm{i}}}{2}\right)^{2}\right] \rho_{\mathrm{I}}-m_{\mathrm{I}}^{\mathrm{H}}(z)\right\}\left(\frac{L}{2}\right)^{2}
$$




\begin{tabular}{ll}
\hline \hline & $L=6.68 \mathrm{~cm}$ \\
tube length & $d_{\mathrm{e}}=0.57 \mathrm{~cm}$ \\
tube external diameter & $d_{\mathrm{i}}=0.40 \mathrm{~cm}$ \\
tube internal diameter & $d_{\mathrm{e}}^{\mathrm{H}}=1.47 \mathrm{~cm}$ \\
head external diameter & $d_{\mathrm{i}}^{\mathrm{H}}=1.41 \mathrm{~cm}$ \\
head internal diameter & $d_{\mathrm{e}}^{\mathrm{B}}=1.79 \mathrm{~cm}$ \\
body external diameter & $d_{\mathrm{i}}^{\mathrm{B}}=1.73 \mathrm{~cm}$ \\
body internal diameter & $h_{\mathrm{u}} \approx 0.3 \mathrm{~cm}$ \\
height of upper spherical calotte (empty) & $\varphi \approx 20^{\circ}$ \\
angle of Fig. 1 & \\
& $m_{\mathrm{h}}=0.81 \mathrm{~g}$ \\
hat mass & $m_{\mathrm{b}} \approx 0.2 \mathrm{~g}$ \\
beak mass & $\rho_{\mathrm{g}}=2.10 \mathrm{~g} \mathrm{~cm}{ }^{-3}$ \\
& $c_{\mathrm{g}}=0.837 \mathrm{~J} \mathrm{~g}^{-1}{ }^{\circ} \mathrm{C}^{-1}$ \\
glass density & \\
glass specific heat & $\rho_{\mathrm{I}}=1.336 \mathrm{~g} \mathrm{~cm}^{-3}$ \\
& $T_{\mathrm{b}}^{\mathrm{I}}=313.15 \mathrm{~K}^{-3}$ \\
$\mathrm{CH}_{2} \mathrm{Cl}_{2}$ density & $\Delta h_{v}^{\mathrm{I}}=28094.50 \mathrm{~J} \mathrm{~mol}^{-1}$ \\
$\mathrm{CH}_{2} \mathrm{Cl}_{2}$ normal boiling point & \\
$\mathrm{CH}_{2} \mathrm{Cl}_{2}$ vaporization enthalpy & \\
\hline
\end{tabular}

Table 2: Geometrical and physical data for our drinking bird [5], whose internal liquid is methylene chloride, $\mathrm{CH}_{2} \mathrm{Cl}_{2}$ (see Fig. 1).

$$
+z \pi \rho_{\mathrm{I}}\left(\frac{d_{\mathrm{i}}}{2}\right)^{2}\left[\frac{1}{12} z^{2}+\left(\frac{L}{2}-\frac{z}{2}\right)^{2}\right]+m_{\mathrm{I}}^{\mathrm{H}}(z)\left(\frac{L}{2}\right)^{2},
$$

where we have used Steiner's theorem. In Eq. (14), $I_{0}$ is the moment of inertia for everything but the internal liquid, $V_{\mathrm{I}}$ is the internal liquid volume

$$
V_{\mathrm{I}}=\frac{4}{3} \pi\left(\frac{d_{\mathrm{i}}^{\mathrm{B}}}{2}\right)^{3}-\frac{1}{3} \pi h_{\mathrm{u}}^{2}\left(3 \frac{d_{\mathrm{i}}^{\mathrm{B}}}{2}-h_{\mathrm{u}}\right)
$$

and $m_{\mathrm{I}}^{\mathrm{H}}(z)$ is the mass of the internal liquid in the head, which depends on $z(t)$ :

$$
m_{\mathrm{I}}^{\mathrm{H}}(z)= \begin{cases}{[z(t)-L] \pi\left(\frac{d_{\mathrm{i}}}{2}\right)^{2} \rho_{\mathrm{I}}} & , \text { if } z(t) \geq L \\ 0 & , \text { if } z(t)<L .\end{cases}
$$

The second term of (14) refers to the liquid that remains in the body, the third term describes the liquid in the tube, and the last term the liquid in the head.

The moment of inertia $I_{0}$ may be written

$$
\begin{aligned}
I_{0} & =m_{\mathrm{g}}^{\mathrm{B}}\left(\frac{L}{2}\right)^{2}+\frac{2}{3} m_{\mathrm{g}}^{\mathrm{B}}\left(\frac{d_{\mathrm{i}}^{\mathrm{B}}}{2}\right)^{2}+\frac{1}{12} m_{\mathrm{t}} L^{2} \\
& +\left(m_{\mathrm{g}}^{\mathrm{H}}+m_{\mathrm{h}}+m_{\mathrm{E}}\right)\left(\frac{L}{2}\right)^{2}+\frac{2}{3} m_{g}^{\mathrm{H}}\left(\frac{d_{\mathrm{i}}^{\mathrm{H}}}{2}\right)^{2}+\left(m_{\mathrm{b}}+m_{\mathrm{b}}^{\mathrm{E}}\right) L_{\mathrm{b}}^{2} .
\end{aligned}
$$


The masses in this equation are: $m_{\mathrm{g}}^{\mathrm{B}}=\left(4 \pi \rho_{\mathrm{g}} / 3\right)\left[\left(d_{\mathrm{e}}^{\mathrm{B}} / 2\right)^{3}-\left(d_{\mathrm{i}}^{\mathrm{B}} / 2\right)^{3}\right]$, mass of glass in body; $m_{\mathrm{g}}^{\mathrm{H}}=\left(4 \pi \rho_{\mathrm{g}} / 3\right)\left[\left(d_{\mathrm{e}}^{\mathrm{H}} / 2\right)^{3}-\left(d_{\mathrm{i}}^{\mathrm{H}} / 2\right)^{3}\right]$ mass of glass in head; $m_{\mathrm{t}}=\pi \rho_{\mathrm{g}}\left[\left(d_{\mathrm{e}} / 2\right)^{2}-\left(d_{\mathrm{i}} / 2\right)^{2}\right]$, mass of the tube; $m_{\mathrm{h}}$, mass of the hat; $m_{\mathrm{E}}$, mass of water on the head except the beak; $m_{\mathrm{b}}$, mass of the beak; and $m_{\mathrm{b}}^{\mathrm{E}}$, mass of water in the beak. In the last term of Eq. (17), which stands for the beak,

$$
L_{\mathrm{b}}^{2}=l_{\mathrm{b}}^{2}\left(1+\operatorname{tg}^{2} \varphi\right)
$$

with $l_{\mathrm{b}} \approx\left(d_{\mathrm{e}}^{\mathrm{H}}+L\right) / 2$ and $\varphi$ the angle shown in Fig. 1. Again, Steiner's theorem was used and, for the glass tube, the rotation axis was considered at the tube's center.

The torque with respect to the fixed rotation axis is given by

$$
\begin{aligned}
M(z)=M_{0} & -\left\{\left[V_{\mathrm{I}}-z \pi\left(\frac{d_{\mathrm{i}}}{2}\right)^{2}\right] \rho_{\mathrm{I}}-m_{\mathrm{I}}^{\mathrm{H}}(z)\right\} \frac{L}{2} g \sin \theta \\
& -\rho_{\mathrm{I}}\left[z \pi\left(\frac{d_{\mathrm{i}}}{2}\right)^{2}\right] \frac{1}{2}(L-z) g \sin \theta+m_{\mathrm{I}}^{\mathrm{H}}(z) \frac{L}{2} g \sin \theta,
\end{aligned}
$$

with $\theta$ the angle between the vertical and the tube. In (19) the moment of the "fixed" parts is

$$
\begin{aligned}
M_{0}= & -m_{g}^{\mathrm{B}} \frac{L}{2} g \sin \theta+\left(m_{\mathrm{g}}^{\mathrm{H}}+m_{\mathrm{h}}+m_{\mathrm{E}}\right) \frac{L}{2} g \sin \theta \\
& +\left(m_{\mathrm{b}}+m_{\mathrm{b}}^{\mathrm{E}}\right) L_{\mathrm{b}} g \sin (\theta+\varphi)
\end{aligned}
$$

\section{Acknowledgments}

This work was partially supported by the Spanish Ministerio de Ciencia y Tecnología (Grant BFM2000-1150) and by the program "Portuguese-Spanish Integrated Actions".

\section{References}

[1] M. V. Sullivan, U.S. Patent 2,402,463 (1946); H. R. Crane, What does the drinking bird know about jet lag, The Physics Teacher 27, 470 (1989); K. B. Kolb, Drinking Duck shutter, The Physics Teacher 5, 342 (1967); L. M. Ng and Y. S. Ng, The thermodynamics of the drinking bird toy, Phys. Educ. 28, 320 (1993); G. K. Vemulapalli, A discourse on the Drinking Bird, J. Chem. Edu. 67, 457 (1990); H. E. Stockman, Dunking Duck without liquid, Am. J. Phys. 29, 335-336 (1961); H. E. Stockman, Secret of the Dunking Duck, Am. J. Phys. 29, 374-375 (1961); E. Guarner and A. M. Sánchez, The superconducting bird: a didactical toy, The Physics Teacher 30, 176-177 (1992);

C. F. Bohren, Clouds in a Glass of Beer, John Wiley, New York, 1987. 
[2] J. S. Miller, Physics of the Dunking Duck, Am. J. Phys. 26, 42-43 (1958). J. L. Gaines, Dunking Duck, Am. J. Phys. 27, 189-190 (1959); D. L. Frank, The drinking bird and the scientific method, J. Chem. Edu. 50, 211 (1973).

[3] R. Plumb, Physical chemistry of the drinking duck, J. Chem. Edu. 50, 212 (1973); K. B. Kolb, Reciprocating engine, The Physics Teacher 4, 121-122 (1966).

[4] R. Mentzer, The drinking bird. The little heat engine that could, The Physics Teacher 31, 126-127 (1993).

[5] Drinking Bird Arora (C)1997. Patent 100616. Tobar Ltd. Norfolk, UK.

[6] J. Güémez, R. Valiente, C. Fiolhais, and M. Fiolhais, Experiments with the sunbird, submitted for publication.

[7] D. A. McQuarrie and J. D. Simon, Molecular Thermodynamics, University Science Books, Sausalito CA (1999); D. V. Schroeder, Introduction to Thermal Physics, Addison-Wesley Publishing (1999); M. W. Zemansky and R. H. Dittman, Heat and Thermodynamics, 7th ed., McGraw Hill, Singapore (1997).

[8] K. Raznjevic, Tables et Diagrammes Thermodynamiques, Editions Eyrolles, Paris (1970)

[9] A. J. Abraham and C. P. Bean, A simple method for measurement of the diffusion of vapors, Am. J. Phys. 57, 325-329 (1989).

[10] J. I. Zubizarreta and G. Pinto, An ancient method for cooling water explained by mass and heat transfer, Chem. Eng. Edu. Spring 95, 96-99 (1995).

[11] H. Gould and J. Tobochnik, An introduction to computer simulation methods, 2nd ed., (Addison-Wesley Publishing Company, Reading, 1996)

[12] C. Bachhuber, Energy from the evaporation of water, Am. J. Phys. 51, 259-264 (1983). 

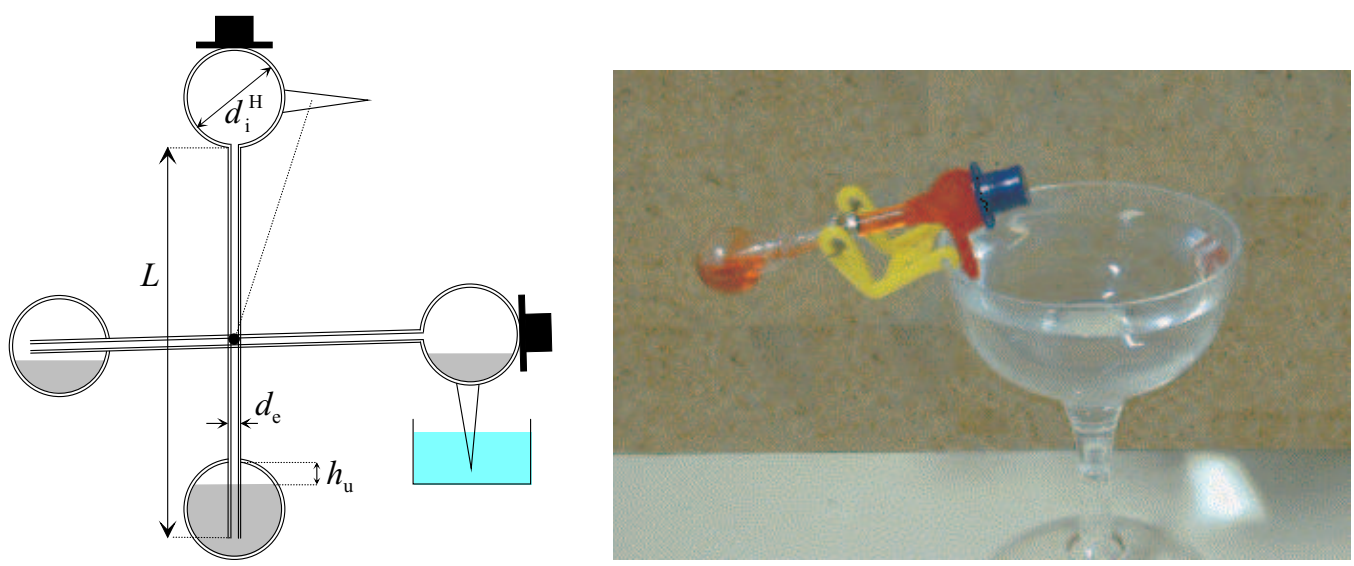

Figure 1: Drinking bird scheme and photo. For dimensions see Tab. 2 in Appendix A.
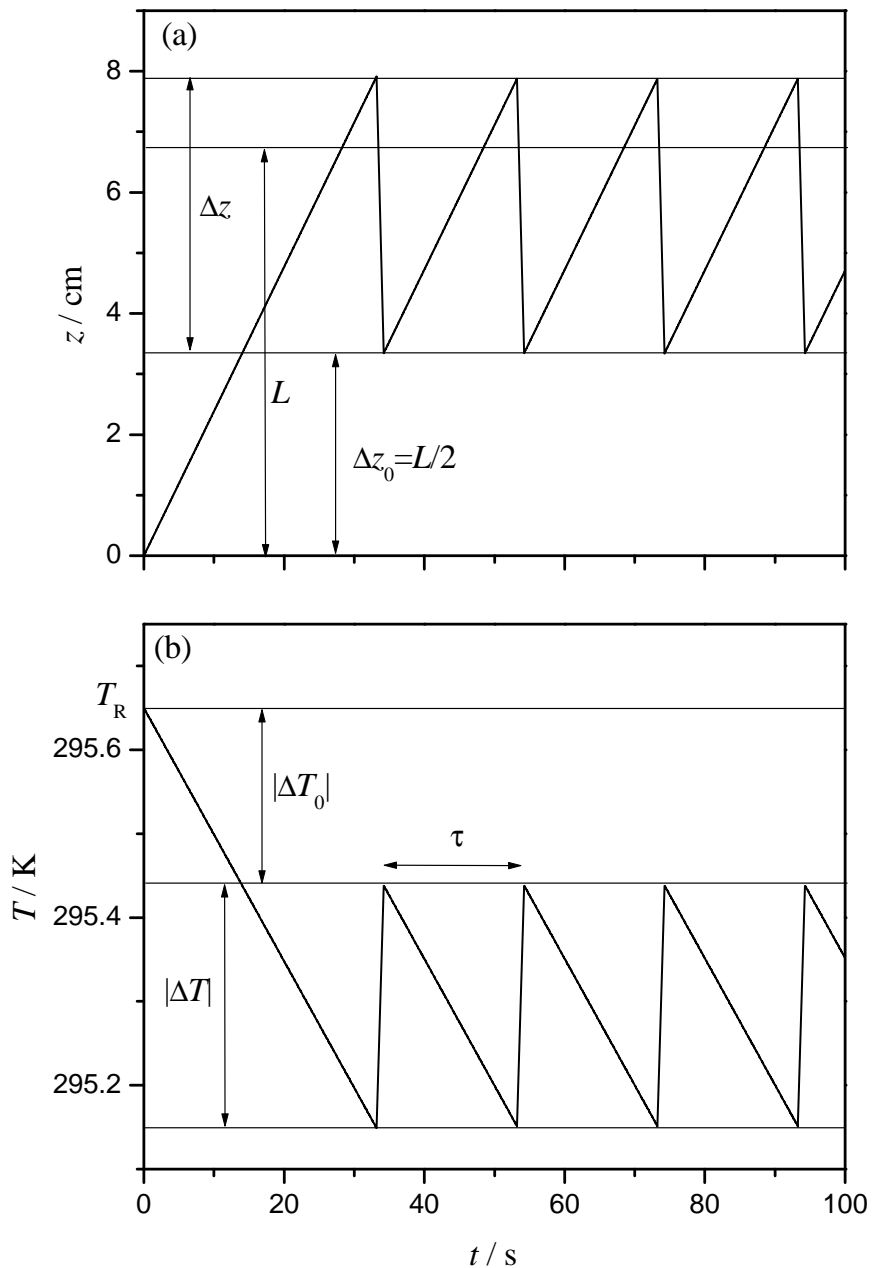

Figure 2: Evolution with time (a) of internal liquid height, $z$, and (b) temperature inside the bird's head, $T$. These results were obtained in the simulation described in Sec. 4, with the parameters as indicated in Fig. 5 (a). 

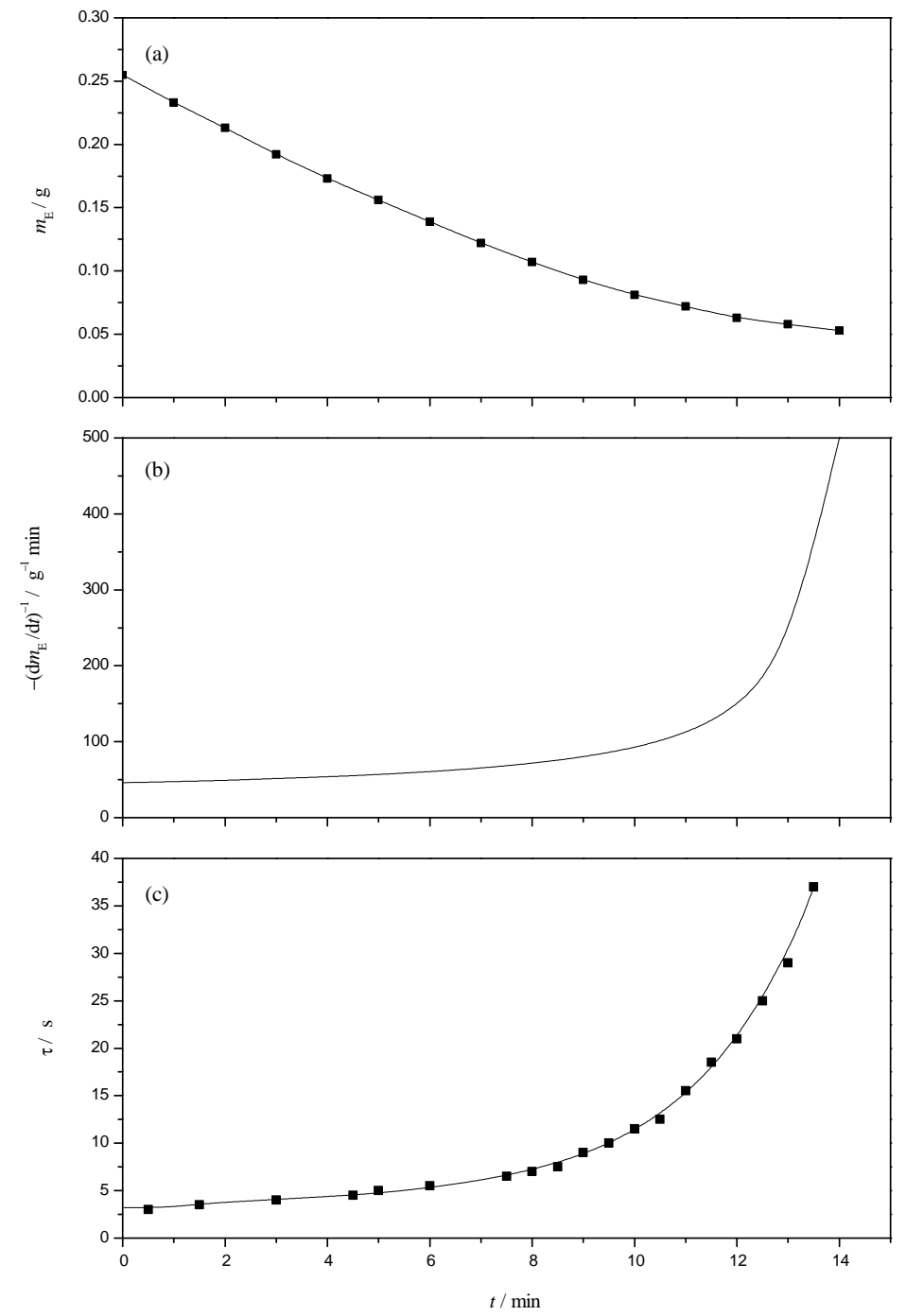

Figure 3: Mass $m_{\mathrm{E}}$ of ethylic alcohol on the bird's head (a), evaporation rate $\dot{m}_{\mathrm{E}}$ obtained from the derivative of the fitting curve to $m_{\mathrm{E}}(\mathrm{b})$, and period $\tau$ (c), as a function of time. For time intervals less than one minute (a few periods, see Tab. 1) the evaporation rate is approximately constant. Similar results are obtained for methylic alcohol, chloroform, and ethyl acetate. n-Hexane evaporates too quickly. For water, the evaporation rate is practically constant in the considered time scale. 

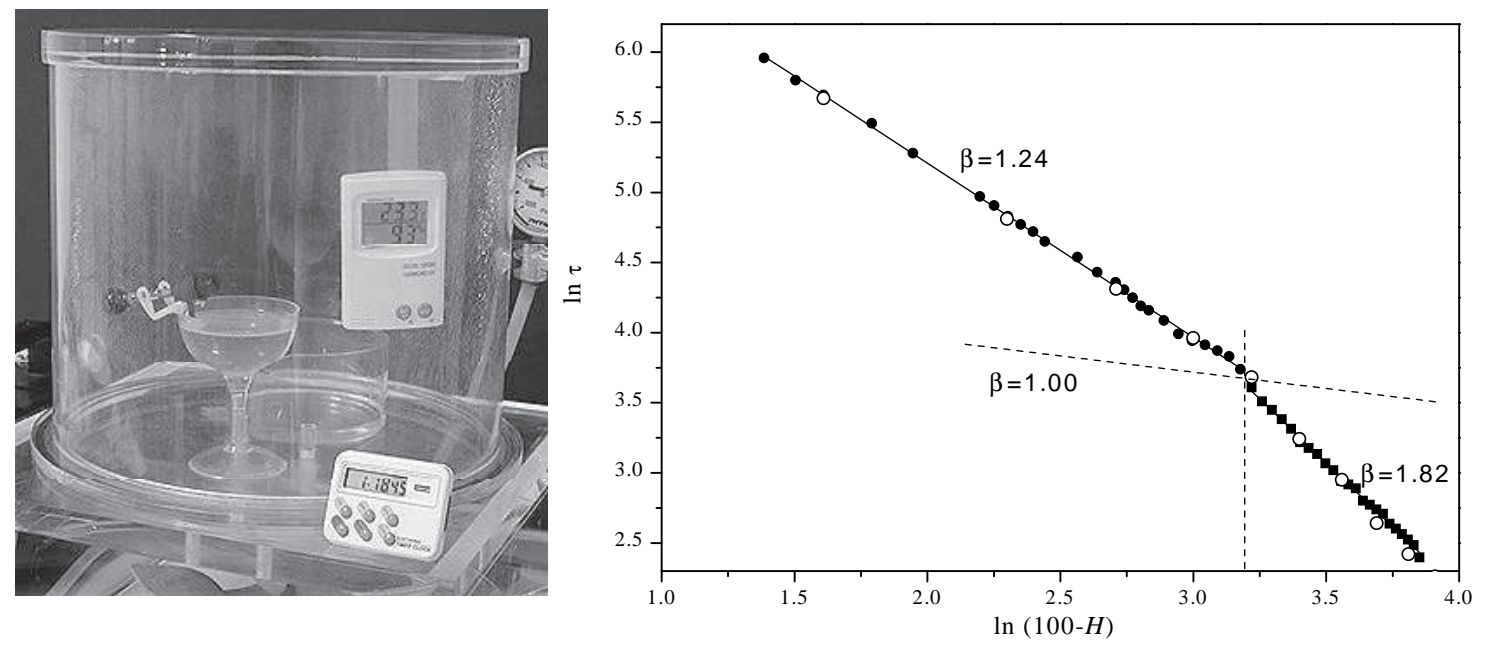

Figure 4: On the left: setup for our experiments on the humidity dependence of the drinking bird period. The bird is placed inside a closed transparent chamber. A digital chronometer and a digital hygrometer complete the set. The humidity, which started around $50 \%$, was increasing during an experiment. On the right: logarithm of the period, $\ln \tau$, versus $\ln (100-H)$. Two different evaporation regimes are observed, one for high (black circles) and the other for low (black squares) humidities. The changing from one regime to the other occurs at $\ln (100-H)=3.2$. Least squares fits yield $\ln \tau=9.44-$ $1.82 \ln (100-H)$ for low humidities and $\ln \tau=7.69-1.24 \ln (100-H)$ for high humidities. The open circles are the results of the simulation presented in Sec. 4. 

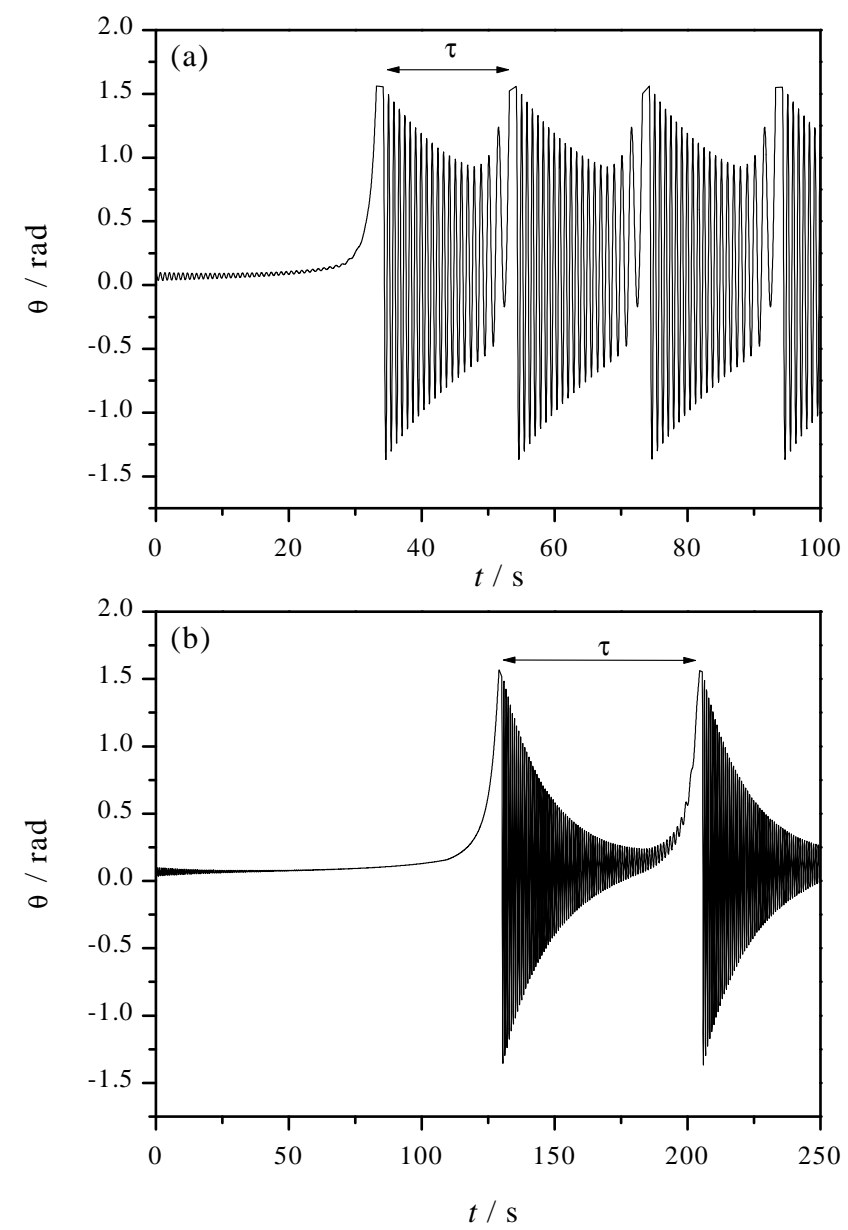

Figure 5: Numerical simulation of the time evolution of the angle for a drinking bird with mass of water in the head $m_{\mathrm{E}}=0.4 \mathrm{~g}$ and mass of water in the beak $m_{\mathrm{b}}^{\mathrm{E}}=0.1$ $\mathrm{g}$ (see Appendix B for usage of these parameters in the moment of inertia and torque) and humidities (a) $H=65 \%$ and (b) $H=85 \%$. The initial conditions are in both cases $\theta(0)=0.1 \mathrm{rad}$ and $\omega(0)=0 \mathrm{rad} / \mathrm{s}$. Note that the time scale is not the same in (a) and (b). The period is almost four times longer for the higher humidity. 